\title{
How to Use the Book
}

In our increasingly digital world writing a book instead of producing a youtube video may appear somewhat strange. However, I am convinced that digital formats such as videos alone, do not yield a long-lasting, in-depth understanding of complicated material. Personally, I learned the most when sitting at home trying to solve a problem which sometimes required several hours of thinking, i. e. it required effort. Everyone who plays a musical instrument knows exactly that the ability to play the instrument does not improve by watching videos or listening to the music played by somebody else; this can at best provide instructions, give guidelines or can be stimulating. Making improvements ultimately requires practice. Reading a book requires effort, because it is an active process and stimulates thinking about the content. In contrast, watching a movie is a rather passive process. In fact, I frequently see my kids writing messages on their cell phones while watching a movie on TV but I never see this when they read a book because they are completely absorbed in the latter case. Apparently, being actively involved in the learning process strongly helps understanding and memorizing the content. From this point of view, a textbook is ideal since the content requires effort and one must be actively involved. On the other hand, every student knows that textbooks can also be highly frustrating in that sometimes the solutions to the problem sets are either missing or the solution is provided in such a reduced way that it is difficult or near impossible to follow. Here, watching a video with step by step instructions is extremely helpful. Furthermore, an appropriate animation is often more elucidating than many pages of written explanations.

The contents of the present book is therefore digitally enhanced in that video explanations and supporting material as well as animations are provided through QRcodes. Moreover, there are a number of tasks integrated into the text that are provided with video solutions. As mentioned above, learning is most effective if you try to solve these problems yourself before watching the solution. To support doing so, the videos start with providing hints regarding the solution before going into the details. 
